\title{
Dietary fish oil and airways obstruction
}

\author{
John Britton \\ University of Nottingham, Division of Respiratory Medicine, City Hospital, \\ Nottingham, UK
}

Introductory article

\section{Dietary n-3 polyunsaturated fatty acids and smoking-related chronic obstructive pulmonary disease}

E Shahar, AR Folsom, SL Melnick, MS Tockman, GW Comstock, V Gennaro, MW Higgins, PD Sorlie,
W-J Ko, M Szklo for the Atherosclerosis Risk in Communities Study Investigators

Background. Fish contain n-3 polyunsaturated fatty acids, principally eicosapentaenoic acid and docosahexaenoic acid, which are known to interfere with the body's inflammatory response and may be of benefit in chronic inflammatory conditions. Methods. We studied the relation between the dietary intake of n-3 fatty acids and chronic obstructive pulmonary disease (COPD) in 8960 current or former smokers participating in a population-based study of atherosclerosis. Intake of fatty acids was estimated with a dietary questionnaire. The presence of COPD was assessed by a questionnaire on respiratory symptoms and by spirometry. Three case definitions of COPD were used: symptoms of chronic bronchitis (667 subjects), physician-diagnosed emphysema reported by the subject (185 subjects), and spirometrically detected COPD (197 subjects). Results. After control for pack-years of smoking, age, sex, race, height, weight, energy intake, and educational level, the combined intake of eicosapentaenoic acid and docosahexaenoic acid was inversely related to the risk of COPD in a quantity-dependent fashion. The adjusted odds ratio for the highest quartile of intake as compared with the lowest quartile was 0.66 for chronic bronchitis (95 percent confidence interval, 0.52 to $0.85 ; P<0.001$ for linear trend across the range of intake values), 0.31 for physician-diagnosed emphysema (95 percent confidence interval, 0.18 to 0.52; $P$ for linear trend, 0.003), and 0.50 for spirometrically detected COPD (95 percent confidence interval, 0.32 to 0.79; $P$ for linear trend, 0.007). Conclusions. A high dietary intake of n-3 fatty acids may protect cigarette smokers against COPD. (N Engl J Med 1994;331:228-33)

Why should fish oil protect against airways disease?

The hypothesis that dietary fish intake may somehow reduce susceptibility to chronic airways obstruction probably originated from the observation that the prevalence of asthma is extremely low amongst Eskimo populations. ${ }^{12}$ Whilst there are clearly many genetic and environmental factors that differentiate traditional Eskimo populations from the urbanised, economically developed societies in which asthma is so much more frequent, the effect of dietary fish intake is one factor that has attracted particular interest. However, a role for fish oils in protecting against asthma, and more recently chronic airways obstruction, has also been suggested as a result of developments in the understanding of the mechanisms of synthesis of prostaglandins, leukotrienes, and related compounds from fatty acids derived from animal and fish sources, and the roles of these compounds in the pathophysiology of lung disease..$^{3-6}$

Known collectively as eicosanoids, the prostaglandins and leukotrienes are potent inflammatory mediators with established roles in a diverse range of diseases. ${ }^{3}$ In humans the principal substrate for eicosanoid production is arachidonic acid, an unsaturated fatty acid which comprises a 20-carbon molecule with four double carbon bonds, the first of which is at the sixth carbon atom from the methyl end of the molecule. The structure of arachidonic acid is thus described in nomenclature as $\mathrm{C}_{20: 4}, \mathrm{n}-6 .{ }^{6}$ The production of eicosanoids by inflammatory cells begins with the release of arachidonic acid from membrane phospholipid by phospholipase enzymes, and subsequent metabolism by cyclooxygenase or lipoxygenase, respectively, into prostaglandin or leukotriene products. ${ }^{3}$ Although arachidonic acid can be synthesised to varying degrees in different animals from other dietary precursors including linoleic 


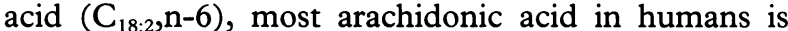
obtained by ingestion of arachidonic acid in animal fats.

Cold water sea fish, and also some mammals that have adapted to life in arctic temperatures, contain more highly polyunsaturated fatty acids than arachidonic acid, particularly eicosapentaenoic acid (EPA, $\left.\mathrm{C}_{20: 5}, \mathrm{n}-3\right)$ and docosahexaenoic acid (DHA, $\left.\mathrm{C}_{22: 6}, \mathrm{n}-3\right)$. Both of these fatty acids act as competitive inhibitors of arachidonic acid metabolism, and also compete to varying degrees with arachidonic acid as a substrate for cyclo-oxygenase or lipoxygenase enzymes to produce eicosanoids with different, and in most cases less, biological activity than their arachidonic acid-derived equivalents. ${ }^{5}$ It therefore follows that a diet which is rich in these n-3 fatty acids should influence and, in general, downregulate the inflammatory potency of endogenous eicosanoids, and thus reduce the prevalence and severity of the diseases to which they contribute.

\section{Role of fish oils in asthma}

The central role played by inflammation of the airways in the pathogenesis of asthma, and in particular the recognition during the early 1980 s that leukotrienes are especially potent mediators of bronchoconstriction, ${ }^{78}$ led to investigations of the effect of $n-3$ acid supplementation in patients with asthma. Although these trials involved relatively short periods of supplementation (the maximum being 10 weeks), their results were not encouraging in that, although EPA supplementation was shown to reduce leucocyte levels of arachidonic acid, to reduce neutrophil chemotaxis and leukotriene generation, and to reduce the airway late response to allergen, ${ }^{9-11}$ none of the trials provided any evidence of clinically appreciable improvements in the severity of asthma. ${ }^{10-12}$ Indeed, one study of patients with aspirin intolerant asthma found that control of asthma deteriorated during fish oil supplementation. ${ }^{13}$ These experimental data do not therefore support the idea that fish oils have a major protective effect in asthma. In terms of epidemiological studies, little has been published on the relation between fish oil intake and the occurrence of asthma, and although a study of the Second National Health and Nutrition Survey (NHANES-II) dataset by Schwartz and Weiss suggested that a high fish intake was significantly associated with a lower occurrence of self reported wheezing, this relation did not persist after adjustment for the effects of other dietary factors including vitamin $\mathrm{C}$ and niacin. ${ }^{14}$ Whether dietary fish oil has any independent effect on the risk of asthma is therefore, on the basis of the available evidence, unresolved.

\section{Role of fish oils in chronic airways obstruction}

Interest in the role of fish oils in the aetiology of lung disease has recently shifted to their potential preventive role in relation to chronic airways obstruction. The rationale for this shift of emphasis lies in the recognition that inflammatory processes in the airway and in the lung parenchyma play an important part in the development of chronic bronchitis and emphysema, which are the principal diseases of chronic airways obstruction, and that inflammation driven by neutrophils plays a major part in this process. ${ }^{15-17}$ Since eicosanoids are mediators of neutrophil-induced inflammation, it is plausible that a high dietary intake of fish oil may reduce the development of chronic airways obstruction through the inhibitory and competitive effects on arachidonic acid metabolism outlined above.

The recent study by Shahar et $a l,{ }^{18}$ in the introductory article which demonstrated a reduced occurrence of chronic obstructive pulmonary disease (COPD) in individuals with a relatively high intake of $n-3$ fatty acids, is one of four studies of the relation between dietary fish oil and either lung function or clinically defined chronic airflow obstruction published within the past two years. ${ }^{18-21}$ Three of these studies were cross sectional, of which the study by Shahar et al was the largest, involving an initial population of 15800 men and women aged 45-64 from four communities taking part in the Atherosclerosis Risk in Communities (ARIC) study. The analysis was primarily restricted, however, to approximately 9000 subjects who had ever been smokers. Four different COPD groups were defined, comprising those with a history of chronic productive cough (cough and phlegm on most mornings for three months of the year for at least two years), those in whom a doctor had diagnosed emphysema, those with a forced expiratory volume in one second $\left(\mathrm{FEV}_{1}\right)$ below $65 \%$ of predicted in conjunction with a forced vital capacity (FVC) above $80 \%$ predicted, and a composite group including all subjects meeting any one of these criteria. The intake of EPA and DHA was estimated from the self reported frequency of consumption of three different types of fish (tuna, dark meat fish, and others) on a semiquantitative food frequency questionnaire. The study suggested that the occurrence of COPD by any of the criteria used was lowest amongst those reporting the highest consumption of fish oil. As might be expected, given that total EPA and DHA intake were estimated from an arithmetic transformation of fish portion data, the relationships with COPD were qualitatively consistent whether fish intake was measured in terms of estimated total EPA and DHA intake or of weekly consumption of fish servings. In general, the overall risk of COPD by any of the definitions used was reduced in the highest relative to the lowest quartile of fish oil intake by an odds ratio, adjusted for smoking, educational level, race, and age, sex, height and weight, of the order of $0 \cdot 3-0 \cdot 6$. There was no appreciable relation between fish oil intake and smoking exposure.

If a low n-3 fatty acid intake is indeed a cause of COPD, then n-3 fatty acid intake should also be inversely related to impairment of lung function. The authors also investigated this relationship and, in this respect, the findings of the paper were rather less consistent. Although an analysis involving the total population was not provided in the paper, data were presented on the association between three indices of lung function $\left(\mathrm{FEV}_{1}, \mathrm{FVC}\right.$, and the $\mathrm{FEV}_{1} / \mathrm{FVC}$ ratio) and both estimated total fatty acid intake and weekly fish serving frequency, in subgroups of subjects categorised according to race, sex, and smoking status. This analysis therefore involved a potential total of 48 statistical hypothesis tests, and it is not clear whether any adjustment for the effects of this multiple hypothesis testing was made. However, associations that were significant at the $5 \%$ level were reported between higher fatty acid 
or fish portion intakes and higher $\mathrm{FEV}_{1}$ and $\mathrm{FEV}_{1} / \mathrm{FVC}$ but not FVC in male white smokers, and $\mathrm{FEV}_{1}, \mathrm{FVC}$, and $F E V_{1} / F V C$ ratio in white female smokers. In these groups the magnitude of the effect was small, being 20-30 $\mathrm{ml}$ for $\mathrm{FEV}_{1}$ between the 25 th and 75 th centiles of dietary intake. In white non-smokers neither measure of fish intake was related significantly to any lung function index, though there was an association of borderline significance between increased fish servings and increased $\mathrm{FEV}_{1}$ in white non-smoking women. The justification for this somewhat extensive subgroup analysis was not presented in the paper, nor were the figures arising from these analyses in the black subgroups.

The initial message that a high intake of fish oil is associated with a decreased risk of COPD amongst smokers must therefore be viewed in the perspective that, while the associations with the binary disease outcomes used by the authors were strong and highly significant, the quantitative associations with lung function were weak and significant only in certain subgroups. In this respect the findings of the paper are inconsistent and, indeed, in view of the multiple hypothesis testing employed in the analysis of lung function data, it is not clear whether any of the observed relationships with lung function were significant at the $5 \%$ level. These are issues which are not addressed by the authors. Other potential weaknesses of the study, which include confounding of fish intake with other protective dietary effects, residual confounding by smoking, selection bias, and possible reverse causation of the relation between lung disease and fish intake, are acknowledged by the authors but are not explored further.

The issues of residual confounding by smoking and of confounding of fish intake with other dietary factors are particularly important. Cigarette smoking is clearly a major risk factor for COPD, as was demonstrated in this study by the fact that spirometrically defined COPD was found in 197 of the 8960 ever smokers and only 20 of the 6456 never smokers. A degree of confounding between smoking and diet could therefore easily lead to an artefactual association between diet and COPD, despite attempts to adjust for smoking in the statistical analysis. The authors report that there was only a very weak association between smoking and fish intake in this study, but it is possible that confounding of these variables and bias arising from systematic errors in reporting both smoking and fish intake may have distorted the true relationship between fish intake and COPD. Confounding of fish intake with other potentially important dietary variables is also particularly relevant since studies of the relation between lung function and other dietary factors such as vitamin $\mathrm{C}$ and magnesium have demonstrated positive associations with lung function that are at least as strong as those described for $\mathrm{n}-3$ fatty acids by Shahar et al. ${ }^{22-25}$ It is therefore possible that the association between low fish intake and COPD arises as a consequence of confounding of low fish intake with these or other potentially relevant nutrients. The dietary questionnaire method used in the ARIC study can provide more extensive nutrient information, but data on dietary confounders were not presented in the published analysis.

If valid, however, the paper by Shahar does suggest that $n-3$ fatty acids may have a beneficial effect in relation to COPD, and two other recent cross sectional studies demonstrating evidence of higher lung function in individuals with a higher fish oil intake provide additional support for this hypothesis. ${ }^{190}$ In an assessment of data from the Honolulu Heart Program, Sharp et $a l^{19}$ analysed the relation between $\mathrm{FEV}_{1}$ and self-reported frequency of fish consumption, categorised as high or low. As in the ARIC study there was again no evidence of an association between fish intake and $\mathrm{FEV}_{1}$ in never smokers and, on the basis of this observation, never smokers were excluded from further analysis. Amongst ever smokers, and after adjustment for smoking, age, and height, $\mathrm{FEV}_{1}$ was lower among ex-smokers with a high fish intake, though not significantly so; among current smokers FEV $_{1}$ was a significantly higher by $47 \mathrm{ml}$ (95\% confidence interval 16 to 79) in those with a high fish intake. The study adopted the spirometric definition of COPD used in the ARIC paper ${ }^{18}$ and found a significantly lower odds of COPD in the high fish intake group. From what was again a fairly extensive subgroup analysis, the authors demonstrated that the major part of the difference in lung function between the high and low fish intake groups was attributable to the effect in persons with a smoking history of more than 40 years.

Interestingly, these subgroup analyses also showed that high fish intake was associated with a lower degree of loss of $\mathrm{FEV}_{1}$ per year of cigarette smoking, but not in relation to pack years or number of cigarettes smoked. Thus these findings suggest that $\mathrm{n}-3$ fatty acids protect against $\mathrm{FEV}_{1}$ loss in longer term smokers, but not necessarily in heavier smokers. This is perhaps inconsistent with a simple model of protection against the adverse effects of cigarette smoking, but is at least broadly consistent with the overall suggestion that smokers with impaired lung function tend to take less fish in their diet. Further evidence that this is the case was also presented recently by Schwartz and Weiss ${ }^{20}$ in a cross sectional analysis of data from 2526 adults taking part in the NHANES-I survey. Their analysis included a thorough assessment of alternative methods of modelling $\mathrm{FEV}_{1}$ and the effects of cigarette smoking and other potential confounders, and provides evidence of an effect of fish intake on $\mathrm{FEV}_{1}$ which was independently significant but very small, with $\mathrm{FEV}_{1}$ differing by approximately $3 \%$ across the range of fish intake. However, in this study the effect of fish oil was not significantly different between smoking categories and was, in fact, slightly greater - though not significantly so - amongst non-smokers.

Only one study to date has addressed the fish oil hypothesis in a prospective data set. In a longitudinal study of the incidence of chronic non-specific lung disease (CNSLD) in relation to diet in the Zutphen cohort published some 18 months earlier than the ARIC paper Miedema et $a l^{1}$ defined CNSLD according to criteria of chronic productive cough and diagnosed emphysema which were broadly compatible with those used in the ARIC study, but which also included asthma. In this paper there was no evidence of any association between n-3 fatty acid intake and the incidence of CNSLD over the 25 year study period. There was, however, an increased risk of CNSLD in association with a high linoleic acid intake. The incidence of CNSLD was also inversely related to fruit intake, consistent with a protective effect from antioxidant vitamins or other nutrients. 


\section{LEARNING POINTS}

\section{* Prostaglandins and leukotrienes are probably involved in the pathogenesis of both asthma and COPD.}

* n-3 fatty acids in fish oil inhibit prostaglandin and leukotriene production from arachidonic acid. * Short-term dietary supplementation of $n-3$ fatty acid can reduce neutrophil inflammatory
function and the late response to allergen in the airway.

* Cross sectional data suggest that lung function is lower and COPD more common amongst people with a low dietary fish intake.

* The "COPD effect" of limited dietary fish oil may be confined to certain subgroups of smokers.

* Longitudinal data from the one relevant investigation do not provide evidence that fish oils protect against lung disease.

* The postulated relationships between limited dietary fish oil and obstructive diseases of the airways may have arisen from confounding by other dietary effects.

So what conclusions can be drawn?

Of the evidence available at the time of writing, the cross sectional analyses by Shahar et al, ${ }^{18}$ Sharp et al, ${ }^{19}$ and Schwarz and Weiss ${ }^{20}$ are all superficially consistent with protection against either a decline in lung function or the occurrence of COPD symptoms or diagnosis by a high fish intake. However, the findings of these studies differ in terms of the subgroups in whom fish oil appears to have the greatest effect, and two of them suggest that the effect is only present in smokers. ${ }^{1819}$ None of these studies has controlled for confounding by other nutrients. The only prospective study to address this relationship found no evidence of protection against the incidence of COPD, ${ }^{21}$ and again provided evidence that other nutrients are likely to be involved. There is therefore a consistent message from all of these studies that dietary factors may have an important influence on the occurrence of COPD, but it is not yet clear whether this effect is specific to fish oil.

Given the potential public health significance of dietary manipulation as a means of preventing COPD, it is clearly important that this hypothesis is tested further. Ideally this should involve a prospective clinical trial of the effect of dietary manipulation on the rate of decline in lung function and the occurrence of airways obstruction, but the logistic constraints of such a study in terms of the relatively long time scale and large numbers of subjects likely to be necessary to measure a dietary effect will be so substantial that such information is only likely to become available in the long term future, if ever. For the time being, therefore, it is particularly important to make the best of the data available, and to that end it would be helpful to know whether the effects of fish oil described in the cross sectional studies listed above $\mathrm{e}^{18-20}$ persist after controlling for the effects of other nutrients. If so, then it might be reasonable to suggest that, in relation to the risk of developing chronic airways obstruction, an increased intake of dietary fish oils (probably in conjunction with a reduction in animal fats) may be desirable. In addition, however, any dietary advice should take account of evidence that magnesium and vitamin $\mathrm{C}$ may also have a protective role, and therefore fresh fruits, vegetables, cereals and pulses should be recommended as sources of these nutrients. The latter recommendation probably also applies for asthma, for which magnesium may also be protective, ${ }^{25}$ and in view of the evidence implicating high sodium intake as a risk factor for asthma ${ }^{14,26-28}$ it is probably also sensible to avoid a high sodium intake. The role of fish oil in relation to asthma is much less clear. Finally, it is important to keep sight of the fact that, as a preventative measure, the cessation and avoidance of smoking remains the primary and paramount objective.

1 Herxheimer H, Schaeffer O. Asthma in Canadian Eskimos. $N$ Engl $f$ Med 1974;291:1419.

2 Kromann N, Green A. Epidemiological studies in the Upernavik District, Greenland. Acta Med Scand 1980;208:401-6.

3 Holtzman MJ. Arachidonic acid metabolism. Implications of biological chemistry for lung function and disease. Am Rev Respir Dis 1991;143: 188-203

4 Editorial. Fish oil. Lancet 1988;i:1081-3.

5 Ritter JM, Taylor GW. Fish oil in asthma. Thorax 1988;43:81-3.

6 Hoffman P, Mest HJ. What about the effects of dietary lipids on endogenous prostanoid synthesis? A state-of-the-art review. Biomed Biochim Acta 1987;7:639-50.

7 Weiss JW, Drazen JM, Coles N, McFadden ER, Weller PF, Corey EJ, et al. Bronchoconstrictor effects of leukotriene $\mathrm{C}$ in humans. Science 1982;216: 196-8.

8 Weiss JW, Drazen JM, McFadden ER, Weller P, Corey EJ, Lewis RA, et al. Airway constriction in normal humans produced by inhalation of leukotriene D. Potency, time course and effect of aspirin therapy. fAMA 1983;249:2814-7.

9 Payan DG, Wong MYS, Chernov-Rogan T, Valone F, Pickett WC Blake VA, et al. Alterations in human leukocyte function induced by ingestion of eicosapentaenoic acid. F Clin Immunol 1986;6:402-10.

10 Arm JP, Horton CE, Mencia-Huerta JM, House F, Eiser NM, Clark $\mathrm{TJH}$, et al. Effect of dietary supplementation with fish oil lipids on mild asthma. Thorax 1988;43:84-92.

11 Arm JP, Horton CE, Spur BW, Mencia-Huerta JM, Lee TH. The effects of dietary supplementation with fish oil lipids on the airways response to inhaled allergen in bronchial asthma. Am Rev Respir Dis 1989;139: $1395-400$

12 Kirsch CM, Payan DG, Wong MYS, Dohlman JG, Blake VA, Petri MA, et al. Effect of eicosapentaenoic acid in asthma. Clin Allergy MA, et al. Effect

13 Picado C, Castillo JA, Schinca N, Pujades M, Ordinas A, Coronas A, et al. Effects of a fish oil enriched diet on aspirin intolerant asthmatic et al. Effects of a fish oil enriched diet on asp

14 Schwartz J, Weiss ST. Dietary factors and their relation to respiratory symptoms. Am $\mathcal{F}$ Epidemiol 1990;132:67-76. 
15 McGowan SE, Hunninghake GW. Neutrophils and emphysema. N Engl f Med 1989;321:968-70.

16 Snider GL. Emphysema: the first two centuries - and beyond. Am Rev Respir Dis 1992;146:1615-22.

17 MacNee W, Wiggs B, Belzberg AS, Hogg JC. The effect of cigarette smoking on neutrophil kinetics in human lungs. N Engl f Med 1989; 321:924-8.

18 Shahar E, Folsom AR, Milnick SL, Tockman MS, Comstock GW, Gennaro V, et al. Dietary n-3 polyunsaturated fatty acids and smokingrelated chronic obstructive pulmonary disease. N Engl $₹$ Med 1994; 331:228-33.

19 Sharp DS, Rodriguez BL, Shahar E, Hwang L, Burchfiel CM. Fish consumption may limit the damage of smoking on the lung. Am $\mathscr{f}$ consumption may limit the damage

20 Schwartz J, Weiss ST. The relationship of dietary fish intake to level of pulmonary function in the first national health and nutrition survey (NHANES I). Eur Respir f 1994;7:1821-4.

21 Miedema I, Feskens EJM, Heederik D, Kromhout D. Dietary determinants of long-term incidence of chronic nonspecific lung disease. The Zutphen Study. Am 7 Epidemiol 1993;138:37-45.

22 Strachan DP, Cox BD, Erzinclioglu SW, Walters DE, Whichelow MJ.
Ventilatory function and winter fresh fruit consumption in a random sample of British adults. Thorax 1991;46:624-9.

23 Schwartz J, Weiss ST. The relationship between dietary vitamin C intake

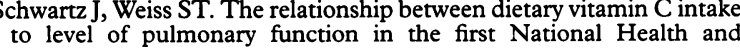
Nutrition Survey (NHANES I). Am $\mathcal{F}$ Clin Nutr 1994;59:110-4.

24 Britton JR, Pavord ID, Richards KA, Knox AJ, Wisniewski AF, Lewis SA. Dietary antioxidant vitamin intake and lung function in the general population Am $\mathcal{F}$ Respir Crit Care Med 1995;151:1383-7.

25 Britton J, Pavord I, Richards K, Wisniewski A, Know A, Lewis S, et al. Dietary magnesium, lung function, wheezing, and airway hyperDeactivity in a random adult population sample. Lancet 1994;344: 357-62.

26 Burney PGJ. The causes of asthma - does salt potentiate bronchial activity? Discussion paper. $\mathcal{f} R$ Soc Med 1987;80:364-7.

27 Burney PGJ, Britton JR, Chinn S, Tattersfield AE, Platt HS, Papacosta $\mathrm{AO}$, et al. Response to inhaled histamine and 24 hour sodium excretion. $B M \mathcal{F}$ 1986;292:1483-6.

28 Pistelli R, Forastiere F, Corbo GM, Dell'Orco V, Brancato G, Agibiti $\mathrm{N}$, et al. Respiratory symptoms and bronchial responsiveness are related to dietary salt intake and urinary potassium excretion in male children. Eur Respir f 1993;6:517-22. 